
\title{
Reseacch S Suare \\ Comparison of 68Ga-DOTA-FAPI and 18FDG PET/CT Imaging Modalities in the Detection of Liver Metastases in Patients with Gastrointestinal System Cancer
}

Ertan Şahin ( $\nabla$ er_ahin@yahoo.com )

Gaziantep University

Umut Elboga

Gaziantep University

Yusuf Zeki Celen

Gaziantep University

Ozlem Nuray Sever

Gaziantep University

Yusuf Burak Cayırlı

Gaziantep University

Ufuk Cimen

Gaziantep University

\section{Research Article}

Keywords: Gastrointestinal system cancer, liver metastasis, 18FDG-PET/CT, 68Ga-DOTATA-FAPI-PET/CT

Posted Date: April 7th, 2021

DOI: https://doi.org/10.21203/rs.3.rs-375922/v1

License: () (i) This work is licensed under a Creative Commons Attribution 4.0 International License. Read Full License 


\section{Abstract}

Purpose

We aimed to compare the diagnostic performance of PET/CT imaging performed with 68Ga-DOTA-FAPI and 18FDG in detection of liver metastases in patients with gastrointestinal system (GIS) cancer.

Methods

A total of 31 patients who underwent ${ }^{68} \mathrm{Ga}$-DOTA-FAPI and ${ }^{18} \mathrm{~F}$-FDG PET/CT examinations and diagnosed with GIS cancer ( 15 colorectal, 9 pancreas, 4 stomach and 3 other cancers) were included in the study. The presence of liver metastasis was decided based on histopathologic diagnosis, $\mathrm{PET} / \mathrm{CT}$, other radiologic examinations or tumor biomarker findings, and both PET/CT imaging findings were compared on the patient and lesion basis.

Results

Of the 31 patients, 28 were found as true positive with ${ }^{68} \mathrm{Ga}$-DOTA-FAPI-PET/CT and 17 with ${ }^{18} \mathrm{FDG}$-PET/CT. Of the 98 metastatic liver lesions determined according to our diagnostic criteria, 92 were found as true positive lesions with ${ }^{68} \mathrm{Ga}$-DOTA-FAPI-PET/CT and 65 with ${ }^{18} \mathrm{FDG}$-PET/CT. There was a statistically significant difference between both imaging modalities in the patient and lesion based comparisons $(p<0.05)$.

When semiquantitative values (SUVmax, mlr) obtained from the lesions were compared between the two imaging methods, mlr values showed statistically significant difference in all tumor subgroups $(p<0.05)$.

Conclusion

It was concluded that ${ }^{68} \mathrm{Ga}$-DOTA-FAPI-PET/CT was superior over ${ }^{18} \mathrm{FDG}$-PET/CT in detection of liver metastases of GIS cancers and it can be a complementary method especially in negative cases with ${ }^{18}$ FDG-PET/CT.

\section{Introduction}

Liver is one of the most critical organs for metastasis in gastrointestinal system cancers and liver metastasis may develop especially in about $70 \%$ of patients with adenocarcinomas [1]. The presence and prevalence of liver metastases is of great importance for treatment options and survival [2].

Radiological imaging modalities (USG, CT, MRI) and ${ }^{18}$ Fluorodeoxyglucose-positron emission tomography/computed tomography $\left({ }^{18} \mathrm{FDG}-\mathrm{PET} / \mathrm{CT}\right)$ methods are commonly used in daily practice in detection of liver metastases and treatment planning $[3,4]$.

${ }^{18} \mathrm{FDG}$ that reflects tumor glucose metabolism is the most widely used PET radiopharmaceutical worldwide. On ${ }^{18}$ FDG-PET/CT imaging, false negative or positive findings may be seen depending on various factors such as the histopathological type, differentiation status, size and glucose metabolic activity of the tumor, or some benign pathologies, infective inflammatory processes, physiological radiopharmaceutical uptake.

Therefore, studies are underway to develop novel radiopharmaceuticals that are more specific to the tumor and have a high target/background ratio. 
Recently, a novel radiopharmaceutical developed on the basis of fibroblast activation protein (FAP) has been increasingly used in tumor diagnosis and staging.

Fibroblast activation protein (FAP) is a membrane glycoprotein highly expressed by cancer-associated fibroblasts (CAFs) seen in cancer types such as colorectal, pancreatic, ovarian and hepatocellular cancers with a strong desmoplastic reaction in which approximately $90 \%$ of gross tumor mass is characterized by stromal content [57].

Due to the relative specificity of FAP to tumor cells, FAP-specific inhibitors (FAPI) have been produced firstly as anticancer drugs and then as tumor-targeted radiopharmaceuticals. [8, 9].

Previous studies have reported that FAPI compound combined with ${ }^{68} \mathrm{Ga}$-DOTA is very practical for diagnosis because of its in vivo positive pharmacokinetic properties and high tumor-background activity in different tumor types on PET imaging [10-12].

Especially due to physiological ${ }^{18}$ FDG uptake in liver; metastases with low FDG uptake or small-size metastases may not be detected on ${ }^{18} \mathrm{FDG}-\mathrm{PET} / \mathrm{CT}$. In this context, it is seen that there are several studies in the literature comparing various radiological methods (CT, MRI) and ${ }^{18} \mathrm{FDG}-\mathrm{PET} / \mathrm{CT}$ in detection of liver metastases of GIS cancers $[13,14]$.

Recently, studies comparing ${ }^{18} \mathrm{FDG}$-PET/CT and ${ }^{68} \mathrm{Ga}$-DOTA-FAPI-PET/CT in various cancer types and primary liver tumors have also been published [15-17]. These studies have reported that GIS physiological radiopharmaceutical uptake and liver ground activity were low in ${ }^{68} \mathrm{Ga}-\mathrm{DOTA}-\mathrm{FAPI}$-PET/CT.

However, looking at the relevant literature, there is no study directly comparing these two PET radiopharmaceuticals ( ${ }^{68} \mathrm{Ga}-\mathrm{DOTA}$-FAPI and ${ }^{18} \mathrm{FDG}$ ) in detecting liver metastases of GIS cancers. Therefore, in this study we aimed to compare these two PET radiopharmaceuticals in the detection of liver metastases in patients with GIS cancers and to show which PET imaging method would be more useful.

\section{Materials And Methods}

\section{Patients}

Institutional board approval was received for this study. A total of 31 patients with 12 being female and 19 male, and aged between 41-85 years old were included in this study. The patients consisted of those newly diagnosed or followed-up with GIS cancer. ${ }^{68} \mathrm{GaDOTA-FAPI-PET} / \mathrm{CT}$ and ${ }^{18} \mathrm{FDG}$-PET/CT were performed to them for staging or re-staging in our clinic. There was at least a two-week interval between two scans and no treatment or interventional procedure was performed in this time.

Patients with primary liver malignancy or another malignancy and those who were using steroids, cirrhosis, pregnant and under 18 years of age were excluded from the study.

\section{Patient preparation and PET/CT imaging protocols}

${ }^{18}$ FDG-PET/CT 
Following fasting for 12 hours, blood glucose level of the 12 patients was analyzed and those with a blood glucose level $<150 \mathrm{mg} / \mathrm{dL}$ were injected with $5 \mathrm{MBq} / \mathrm{kg}$ intravenous (iv) ${ }^{18} \mathrm{FDG}$.

Sixty minutes after the injection, PET/CT scan was performed with GE Discovery IQ; BGO, 5 Ring, 16 Slice combined PET/CT device (General Electric Company, Milwaukee, Wisconsin, USA) in our clinic. PET/CT scan was performed as whole body imaging from the vertex to the upper part of the femur.

\section{${ }^{68}$ GaDOTA-FAPI-PET/CT}

${ }^{68} \mathrm{Ga}$ was obtained from a ${ }^{68} \mathrm{Ge} /{ }^{68} \mathrm{G}$ a radionuclide generator, combined with DOTA-FAPI compound in the modular laboratory in our center and ${ }^{68} \mathrm{Ga}$-DOTA-FAPI was synthesized (Modular lab-Easy, Eckert \& Ziegler). At the end of the 60-minute preparation process, 60-70\% radiochemical yield was obtained. Control by TLC, HPLC revealed that the radiochemical purity was more than $98 \%$. The synthesized ${ }^{68} \mathrm{Ga}-\mathrm{DOTA}-\mathrm{FAPI}$ was injected to the patient as iv at a dose of $2-3 \mathrm{MBq} / \mathrm{kg}$.

Forty-five minutes after the injection, PET/CT scan was performed with GE Discovery IQ; BGO, 5 Ring, 16 Slice, combined PET/CT device (General Electric Company, Milwaukee, Wisconsin, USA) in our clinic. PET/CT scan was performed as whole body imaging from the vertex to the upper part of the femur.

\section{Evaluation of the Images and Patient-Lesion Analysis}

PET/CT images were evaluated independently by 3 experienced nuclear medicine specialists on the patient and lesion basis.

The diagnosis was confirmed histopathologically only in 5 of 31 patients, while the decision was made according to imaging methods (PET/CT and other radiological methods) and tumor biomarker findings (CA19-9, CEA, AFP, CA125) in 26 patients. While the lesion positivity was considered as "making a decision in favour of metastatic lesion finding in the liver in PET/CT images at least by 2 nuclear medicine specialists" and "elevation of tumor biomarkers in addition to liver metastasis findings with radiological imaging methods", the other situations were considered as lesion negative.

The radiopharmaceutic uptake areas higher than liver parenchyma were quantified with SUVmax and mlr (metastatic lesion/liver uptake ratio) and accepted as positive lesion (liver metastasis) finding in also both PET/CT imaging methods. SUVmax and mlr values of the lesions that were considered positive according to our diagnostic criteria, but no positive findings were detected in PET/CT images, were calculated by re-evaluation of the images.

Imaging findings obtained from both PET/CT methods were compared on the patient and lesion basis.

\section{Statistical Analysis}

Radiological, histopathological and biochemical data of the patients were obtained from the computer registry system (electronic health records) of our hospital, and PET/CT images from the image archive in our clinic.

Data were statistically analyzed with SPSS 22.0 statistical analysis software.

Age, gender and sensitivity were expressed with mean, standard deviation and percentage descriptive statistics. 
Normally distributed quantitative variables were expressed as mean \pm standard deviation (SD) and non-normally distributed variables with [IQR, interquartile range].

The comparisons of PET imaging modalities for detecting patients and lesions were made with McNemar test, while comparisons of SUVmax and mlr values with Wilcoxon signed rank test.

For all statistical tests, $p<0.05$ values were considered statistically significant.

\section{Results}

A total of 31 patients diagnosed with GIS cancer (15 colorectal, 9 pancreas, 4 gastric, 3 other) were included in the study. Of the patients, 19 were male and 12 were female. Of the 31 patients, 9 were newly diagnosed and underwent PET/CT for staging, while 22 patients underwent PET/CT at follow-up after treatment. The mean age of the patients was $61.9 \pm 10.9$ (range; $41-85$ ) years old.

\section{Patient based comparison of PET/CT imaging modalities for the presence of liver metastasis}

${ }^{68} \mathrm{GaDOTA}$-FAPI-PET/CT imaging finding was positive in 29 of 31 patients, while no pathologic finding in favour of liver metastasis was found in 2 patients. If we consider them according to our diagnostic criteria; while 28 of 29 positive patients were true positive patients (12 colorectal, 7 pancreatic, 3 gastric, 6 other cancers), one of 2 negative patients was false negative patient who had gastric cancer.

${ }^{18}$ FDG-PET/CT imaging finding was positive in 22 of 31 patients, while no pathologic finding in favour of liver metastasis was found in 9 patients. According to our diagnostic criteria; while 17 of 22 positive patients were true positive patients (10 colorectal, 2 pancreatic, 2 gastric, 6 other cancers), two of 9 negative patients were true negative patients ( 1 colorectal and 1 pancreatic cancer).

${ }^{68} \mathrm{GaDOTA}$-FAPI-PET/CT showed positive findings in 8 true positive patients for whom liver metastasis was not observed in ${ }^{18} \mathrm{FDG}$-PET/CT imaging (Fig. 1). And conversely, ${ }^{18} \mathrm{FDG}-\mathrm{PET} / \mathrm{CT}$ showed positive finding in 1 true positive patient who had no pathologic findings in ${ }^{68} \mathrm{GaDOTA-FAPI-PET/CT} \mathrm{(Fig.} \mathrm{2).} \mathrm{On} \mathrm{the} \mathrm{other} \mathrm{hand,} \mathrm{no} \mathrm{positive}$ findings were found with either PET/CT imaging methods in a true positive patient with gastric cancer.

According to our diagnostic criteria; patient based sensitivity of ${ }^{68} \mathrm{GaDOTA-FAPI-PET} / \mathrm{CT}$ and ${ }^{18} \mathrm{FDG}$-PET/CT imaging methods in revealing liver metastases were found as $96.6 \%$ and $70.8 \%$, respectively. More positive patients were detected in ${ }^{68} \mathrm{GaDOTA}$-FAPI-PET/CT compared to ${ }^{18} \mathrm{FDG}$-PET/CT, and this difference was statistically significant $(p<0.05 ; 0.039)$.

Patient based comparison of the both imaging modalities is given in Table 1. 
Table 1

Patient based comparison of PET/CT imaging modalities

\begin{tabular}{|c|c|c|c|c|}
\hline & $\begin{array}{l}{ }^{18} \mathrm{FDG}-\mathrm{PET} / \mathrm{CT}(+) \\
(\mathrm{n})\end{array}$ & $\begin{array}{l}{ }^{18} \text { FDG-PET/CT (-) } \\
\text { (n) }\end{array}$ & $\begin{array}{l}\text { Total } \\
\text { (n) }\end{array}$ & p \\
\hline $\begin{array}{l}{ }^{68} \mathrm{GaDOTA-FAPI-PET/CT} \mathrm{(+)} \\
\text { (n) }\end{array}$ & 21 & 8 & 29 & $<0.05$ \\
\hline $\begin{array}{l}{ }^{68} \mathrm{GaDOTA-FAPI-PET} / \mathrm{CT}(-) \\
\text { (n) }\end{array}$ & 1 & 1 & 2 & \\
\hline $\begin{array}{l}\text { Total } \\
\text { (n) }\end{array}$ & 22 & 9 & 31 & \\
\hline n: Patient number, p:McNema & Test & & & \\
\hline
\end{tabular}

\section{Lesion based comparison of PET/CT imaging modalities for the presence of liver metastasis}

A total of 98 liver lesions were found according to our diagnostic criteria in 31 patients included in the study.

A total of 94 liver metastatic lesion findings were found on ${ }^{68} \mathrm{GaDOTA-FAPI-PET/CT}$ imaging. Of these 94 positive lesions, 92 were true positive lesions. Three true metastatic lesions that were determined according to our diagnostic criteria were found in 1 patient who was false negative in ${ }^{68} \mathrm{GaDOTA-FAPI-PET} / \mathrm{CT}$ imaging.

A total of 78 liver metastatic lesion findings were found on ${ }^{18}$ FDG-PET/CT imaging. Of these 78 positive lesions, 65 were true positive lesions. There were a total of 16 true positive metastatic lesions that were determined according to our diagnostic criteria in 7 patients who were false negative on ${ }^{18}$ FDG-PET/CT imaging.

Fifteen true positive lesions that could not be found on ${ }^{18} \mathrm{FDG}-\mathrm{PET} / \mathrm{CT}$ imaging were detected with ${ }^{68} \mathrm{Ga-DOTA}$ FAPI-PET/CT imaging, and conversely 2 true lesions that could not be found on ${ }^{68} \mathrm{Ga}-\mathrm{DOTA}$-FAPI-PET/CT were detected with ${ }^{18}$ FDG-PET/CT.

According to our diagnostic criteria, lesion based sensitivity of ${ }^{68} \mathrm{GaDOTA-FAPI-PET} / \mathrm{CT}$ and ${ }^{18} \mathrm{FDG}-\mathrm{PET} / \mathrm{CT}$ imaging methods in detecting liver metastases were found as $96.8 \%$ and $80.2 \%$, respectively.

The number of detected positive metastatic liver lesions was statistically significantly higher on ${ }^{68} \mathrm{GaDOTA-FAPI-}$ PET/CT, compared to ${ }^{18}$ FDG-PET/CT $(p<0.05 ; 0.001)$. On the other hand, lesion based comparison of both PET/CT imaging methods for each patient did not show statistically significant difference $(p>0.05)$.

Lesion based comparison of the both imaging modalities is given in Table 2. 
Table 2

Lesion based comparison of PET/CT imaging modalities

\begin{tabular}{|lllll|}
\hline & ${ }^{18}$ FDG-PET/CT (+) & ${ }^{18}$ FDG-PET/CT (-) & Total & p \\
& $(n)$ & $(n)$ & $(n)$ & value \\
\hline $\begin{array}{l}{ }^{68} \text { GaDOTA-FAPI-PET/CT (+) } \\
(n)\end{array}$ & 75 & 19 & 94 & $<0.05$ \\
\hline $\begin{array}{l}{ }^{68} \text { GaDOTA-FAPI-PET/CT (-) } \\
\text { (n) }\end{array}$ & 3 & & 4 & \\
\hline $\begin{array}{l}\text { Total } \\
\text { (n) }\end{array}$ & 78 & 1 & 98 & \\
\hline n: Lesion number, p:McNemar Test & 20 & & \\
\hline
\end{tabular}

Comparison of semiquantitative values (SUVmax, mlr) obtained from the metastatic lesions in the both PET/CT methods in tumor groups

No statistically significant difference was found in tumor subgroups between both PET/CT imaging modalities in terms of SUVmax values, while there was a significant difference in favor of ${ }^{68} \mathrm{Ga}$-FAPI-PET-CT for all tumor subgroups in terms of mlr values.

Comparison of both imaging modalities in all tumor subgroups in terms of semiquantitative values (SUVmax, mIr) are shown in Table 3.

Table 3

Comparison of both PET/CT imaging modalities among tumor subgroups in terms semiquantitative (SUVmax, $\mathrm{m} / \mathrm{r}$ ) values

\begin{tabular}{|c|c|c|c|c|c|c|c|c|c|c|}
\hline \multirow{2}{*}{$\begin{array}{l}\text { Tumor } \\
\text { types }\end{array}$} & \multicolumn{4}{|c|}{${ }^{68} \mathrm{GaDOTA-FAPI-PET/CT}$} & \multicolumn{4}{|c|}{${ }^{18} \mathrm{FDG}-\mathrm{PET} / \mathrm{CT}$} & \multirow{2}{*}{$\begin{array}{l}\text { p } \\
\text { value } \\
\text { SUV } \\
\text { median }\end{array}$} & \multirow{2}{*}{$\begin{array}{l}\mathrm{p} \\
\text { value } \\
\mathrm{mlr} \\
\text { range }\end{array}$} \\
\hline & $\begin{array}{l}\text { SUV } \\
\text { median }\end{array}$ & $\begin{array}{l}\text { SUV } \\
\text { range }\end{array}$ & $\begin{array}{l}\text { mlr } \\
\text { median }\end{array}$ & $\begin{array}{l}\text { mlr } \\
\text { range }\end{array}$ & $\begin{array}{l}\text { SUV } \\
\text { median }\end{array}$ & $\begin{array}{l}\text { SUV } \\
\text { range }\end{array}$ & $\begin{array}{l}\text { mlr } \\
\text { median }\end{array}$ & $\begin{array}{l}\text { mlr } \\
\text { range }\end{array}$ & & \\
\hline Colorectal & 5.5 & $\begin{array}{l}0.9- \\
14.8\end{array}$ & 4.5 & $\begin{array}{l}0.6- \\
11.9\end{array}$ & 5.0 & $\begin{array}{l}1.9- \\
12.4\end{array}$ & 1.3 & $\begin{array}{l}0.3- \\
4.1\end{array}$ & 0.25 & 0,00 \\
\hline Pancreas & 5.2 & $\begin{array}{l}1.2- \\
15.4\end{array}$ & 4.1 & $\begin{array}{l}0.9- \\
14.1\end{array}$ & 4.6 & $\begin{array}{l}2.5- \\
7.1\end{array}$ & 1.2 & $\begin{array}{l}0.7- \\
2.4\end{array}$ & 0,60 & 0,00 \\
\hline Gastric & 7.7 & $\begin{array}{l}1.8- \\
18.6\end{array}$ & 6.8 & $\begin{array}{l}1.1- \\
16.7\end{array}$ & 4.5 & $\begin{array}{l}2.0- \\
7.9\end{array}$ & 1.2 & $\begin{array}{l}0.8- \\
2.3\end{array}$ & 0,11 & 0,04 \\
\hline Others & 7.8 & $\begin{array}{l}2.3- \\
13.7\end{array}$ & 5.2 & $\begin{array}{l}2.8- \\
10.4\end{array}$ & 5.0 & $\begin{array}{l}4.3- \\
10.2\end{array}$ & 1.5 & $\begin{array}{l}1.2- \\
3.4\end{array}$ & 0.13 & 0,04 \\
\hline
\end{tabular}




\section{Discussion}

As is known, liver metastases may often develop in GIS cancers, and the presence of metastasis of paramount importance in terms of treatment options and prognosis. As in many tumor groups, PET/CT is a globally accepted hybrid molecular imaging method in the diagnosis, staging and restaging of GIS cancers. ${ }^{18} \mathrm{FDG}$, a glucose analog, is the most commonly used radiopharmaceutical in PET imaging owing to its physical features and practicality in many tumor groups. There are numerous studies comparing ${ }^{18} \mathrm{FDG}$-PET/CT with radiological imaging modalities especially such as MRI in primary and metastatic liver tumors, and these studies have reported some limitations about ${ }^{18}$ FDG-PET/CT $[13,14]$.

The more specific radiopharmaceuticals are also needed in liver tumors as in most tumor subgroups in PET imaging. Recently, it is seen that the studies have been conducted to detect various cancers and metastases with ${ }^{68} \mathrm{GaDOTA}-\mathrm{FAPI}$ which is a novel PET radiopharmaceutical [15-17]. To our knowledge, our study is the first in the literature comparing ${ }^{68} \mathrm{GaDOTA-FAPI-PET} / \mathrm{CT}$ and ${ }^{18} \mathrm{FDG}$-PET/CT methods in detection of liver metastases of GIS cancers. Because of the ${ }^{68} \mathrm{GaDOTA}-\mathrm{FAPI}$ has been introduced as a new radiopharmaceutical in our clinic, this is a pilot study. On the other hand, although the number of the patients is low especially for tumor subgroups, data presented with total numbers of patients and lesions are sufficient for statistical significance.

According to our patient based results; a higher rate of cases was detected with ${ }^{68} \mathrm{GaDOTA-FAPI-PET} / \mathrm{CT}$ compared to those found with ${ }^{18} \mathrm{FDG}$-PET/CT modality and the difference was statistically significant. We believe that lower radiopharmaceutic uptake of liver parenchyma in ${ }^{68} \mathrm{GaDOTA-FAPI-PET/CT}$ is one of the most important factors for this result. Although it was not exactly similar to our study in terms of patient groups and study design, in a study by Chen et al. [16] comparing both modalities, ${ }^{68} \mathrm{GaDOTA-FAPI-PET/CT}$ was found to be superior in detection of the cases and lesions, similarly.

In addition to the advantage provided by ${ }^{68} \mathrm{GaDOTA-FAPI}$ in terms of radiopharmaceutic uptake of liver parenchyma, previous studies have stated that ${ }^{68} \mathrm{GaDOTA}$-FAPI lacks physiological gastric and intestines radiopharmaceutic uptakes that are seen with ${ }^{18} \mathrm{FDG}$, and thus primary focus and peritoneal-mesenteric-omental metastases can be better detected in GIS cancers $[15,16,18]$.

We believe that ${ }^{68} \mathrm{GaDOTA}$-FAPI-PET/CT may be preferred instead of ${ }^{18} \mathrm{FDG}-\mathrm{PET} / \mathrm{CT}$ in GIS cancers in the near future, due to its' superiorities stated in the mentioned studies and its success in detecting liver metastases as demonstrated in our study.

While a significantly higher number of lesions were detected with ${ }^{68} \mathrm{GaDOTA-FAPI-PET} / \mathrm{CT}$ compared to ${ }^{18} \mathrm{FDG}$ $\mathrm{PET} / \mathrm{CT}$ in total lesions number, no statistically significant difference was found for lesion numbers on each-one patient.

Although histopathological confirmation and distinguishing of the differentiation status could not be made in all lesions, as stated before this situtation can be explained with better detection of the lesions because of low liver parenchymal radiopharmaceutical activity. Furthermore, in addition to the high radiopharmaceutic uptake of liver parenchyma in ${ }^{18}$ FDG-PET/CT imaging, especially small-sized lesions might have not been distinguished due to partial volume effect. Other conditions that may explain this results are other processes that may cause false positive and negative findings in ${ }^{18}$ FDG-PET/CT imaging. 
On the other hand, some studies have reported that processes related to inflammation may also cause false positive findings in ${ }^{68} \mathrm{GaDOTA-FAPI-PET} / \mathrm{CT}$ imaging [18-20]. In the considering of the possible causes stated in these studies, cirrhotic patients were excluded from our study in order to minimize the processes that could resulted in inflammation and fibrosis in the liver parenchyma. And also, the decision for positive-negative lesions on the both PET/CT imaging was made taken into account mlr values in addition of SUVmax values to optimize inflammation that could be resulted from hepatosteatosis. In our study, it was found that false positivity in 1 patient and false negativity in 1 patient on ${ }^{68} \mathrm{GaDOTA-FAPI-PET/CT}$.

Unfortunately, since majority of the metastatic lesions could not be confirmed histopathologically and studies in the literature on this subjects have just started to be published, we could not made a clear interpretation about the other possible mechanisms that might have caused false positive-negative results in ${ }^{68} \mathrm{GaDOTA-FAPI-PET/CT}$. Therefore, further studies are needed on this subject in which the lesions are confirmed histopathologically.

In the current study, SUVmax values of the lesions were also found to be in ${ }^{68} \mathrm{Ga}$-FAPI-PET-CT higher than ${ }^{18} \mathrm{FDG}$ $\mathrm{PET} / \mathrm{CT}$, and these results are consistent with the results of Chen et al. [18]. But, no statistically significant difference was found between the two PET/CT imaging modalities in terms of the SUVmax of the lesions in tumor subgroups.

On the other hand, statistically significant difference was found in favor of ${ }^{68} \mathrm{Ga}$-FAPI-PET-CT for mlr values in all subgroups. For further studies that will compare both PET/CT imaging methods, we think that mlr would provide a significant contribution for the detection of liver lesions, and will be useful especially in the cases where making a decision based on SUVmax values is challenging.

The main limitations in our study can be listed as; the number of cases was relatively small especially in tumor subgroups in spite of the sufficient total number of lesions, it was not designed as a prospective randomized study, and not all lesions were histopathologically confirmed.

In conclusion; despite the main limiting factors, our study showed that ${ }^{68} \mathrm{GaDOTA-FAPI-PET/CT}$ is a superior imaging modality over ${ }^{18} \mathrm{FDG}-\mathrm{PET} / \mathrm{CT}$ in detection of liver metastases of GIS cancers, and in current conditions it may be a complementary method for ${ }^{18} \mathrm{FDG}$-PET/CT. We believe that ${ }^{68} \mathrm{GaDOTA-FAPI-PET/CT}$ will be a more practical method in the diagnosis, staging and restaging of GIS cancers because of both its success in detection of liver metastases and the advantages that it provides in visualization of the primary lesions and intra-abdominal metastases, and will take place in diagnosis and follow-up algorithm in line with the results of further studies to be conducted in this field.

\section{Declarations}

Conflict of interest: All authors declare that they have no conflict of interest.

Ethical approval: Institutional board approval was received for this study.

Funding: There is no funding.

\section{Authors' contributions}


All authors contributed to the study conception and design. Material preparation, data collection and analysis were performed by Ertan Şahin, Yusuf Burak Çayırlı. The first draft of the manuscript was written by Ertan Şahin and all authors commented on previous versions of the manuscript. All authors read and approved the final manuscript.

\section{References}

1. Manfredi S, Lepage C, Hatem C, Coatmeur O, Faivre J, Bouvier AM. Epidemiology and management of liver metastases from colorectal cancer. Ann Surg 2006;244(2):254-9. doi:10.1097/01.sla.0000217629.94941.cf

2. Zhang S, Gao F, Luo J, Yang J. Prognostic factors in survival of colorectal cancer patients with synchronous liver metastasis. Colorectal Dis 2010;12(8):754-61. doi:10.1111/j.1463-1318.2009. 01911.x

3. Niekel MC, Bipat S, Stoker J. Diagnostic imaging of colorectal liver metastases with CT, MR imaging, FDG PET, and/or FDG PET/CT: a meta-analysis of prospective studies including patients who have not previously undergone treatment. Radiology 2010;257(3):674-84. doi:10.1148/radiol.10100729

4. Holzapfel K, Reiser-Erkan C, Fingerle AA, Erkan M, Eiber MJ, Rummeny EJ, et al. Comparison of diffusionweighted MR imaging and multidetector-row CT in the detection of liver metastases in patients operated for pancreatic cancer. Abdom Imaging 2011;36(2):179-84. doi:10.1007/ s00261-010-9633-5

5. Siveke JT. Fibroblast-activating protein: targeting the roots of the tumor microenvironment. J Nucl Med 2018;59(9):1412-4.

6. Garin-Chesa P, Old LJ, Rettig WJ. Cell surface glycoprotein of reactive stromal fibroblasts as a potential antibody target in human epithelial cancers. Proc Natl Acad Sci USA 1990;1990(87):7235-9.

7. Hamson EJ, Keane FM, Tholen S, Schilling O, Gorrell MD. Understanding fibroblast activation protein (FAP): substrates, activities, expression and targeting for cancer therapy. Proteomics Clin Appl 2014;8(5-6):454-63.

8. Jansen K, Heirbaut L, Cheng JD, Joossens J, Ryabtsova O, Cos P, et al. Selective inhibitors of fibroblast activation protein (FAP) with a (4-quinolinoyl)-glycyl-2-cyanopyrrolidine scaffold. ACS Med Chem Lett 2013;4:491-6.

9. Loktev A, Lindner T, Mier W, Debus J, Altmann A, Jäger D, et al. A tumor-imaging method targeting cancer associated fibroblasts. J Nucl Med. 2018;59:1423-9.

10. Lindner T, Loktev A, Altmann A, Giesel F, Kratochwil C, Debus J, et al. Development of quinoline-based theranostic ligands for the targeting of fibroblast activation protein. J Nucl Med 2018;59(9): 1415-22.

11. Giesel FL, Kratochwil C, Lindner T, Marschalek MM, Loktev A, Lehnert W, et al. 68Ga-FAPI PET/CT: biodistribution and preliminary dosimetry estimate of 2 DOTA-containing FAP-targeting agents in patients with various cancers. J Nucl Med 2019;60(3): 386-92.

12. Kratochwil C, Flechsig P, Lindner T, Abderrahim L, Altmann A, Mier W, et al. 68Ga-FAPI PET/CT: tracer uptake in 28 different kinds of cancer. J Nucl Med 2019;60(6):801-5. 
13. Eiber M, Fingerle AA, Brugel M, Gaa J, Rummeny EJ, Holzapfel K. Detection and classification of focal liver lesions in patients with colorectal cancer: retrospective comparison of diffusion-weighted MR imaging and multi-slice CT. Eur J Radiol 2012;81(4):683-91. doi:10.1016/j.ejrad. 2011.01.072

14. Niekel MC, Bipat S, Stoker J. Diagnostic imaging of colorectal liver metastases with CT, MR imaging, FDG PET, and/or FDG PET/CT: a meta-analysis of prospective studies including patients who have not previously undergone treatment. Radiology 2010;257(3):674-84. doi:10.1148/radiol.10100729

15. Kratochwil C, Flechsig P, Lindner T, Abderrahim L, Altmann A, Mier W. 68Ga-FAPI PET/CT: tracer uptake in 28 different kinds of cancer. J Nucl Med 2019;60(6):801-5.

16. Chen H, Pang Y, Wu J, Zhao L, Hao B, Wu J, et al. Comparison of [68Ga]Ga-DOTA-FAPI-04 and [18F] FDG $\mathrm{PET} / \mathrm{CT}$ for the diagnosis of primary and metastatic lesions in patients with various types of cancer. Eur $\mathrm{J}$ Nucl Med Mol Imaging 2020;47:1820-32. doi: 10.1007/s00259-020-04769-z.

17. Shi X, Xing H, Yang X, Li F, Yao S, Congwei J, et al. Comparison of PET imaging of activated fibroblasts and ${ }^{18} \mathrm{~F}-\mathrm{FDG}$ for diagnosis of primary hepatic tumours: a prospective pilot study. Eur J Nucl Med Mol Imaging 2020 Oct 24. doi: 10.1007/s00259-020-05070-9. Epub ahead of print.

18. Chen H, Zhao L, Ruan D, Pang Y, Hao B, Dai Y, et al. Usefulness of [68Ga]Ga-DOTA-FAPI-04 PET/CT in patients presenting with inconclusive [18F]FDG PET/CT findings. Eur J Nucl Med Mol Imaging 2021 Jan;48(1):73-86. doi: 10.1007/s00259-020-04940-6.

19. Luo Y, Pan Q, Zhang W. IgG4-related disease revealed by (68)GaFAPI and (18)F-FDG PET/CT. Eur J Nucl Med Mol Imaging 2019;46(12):2625-6.

20. Luo Y, Pan Q, Zhang W, Li F. Intense FAPI uptake in inflammation may mask the tumor activity of pancreatic cancer in 68Ga-FAPI PET/CT. Clin Nucl Med 2020;45(4):310-1.

\section{Figures}
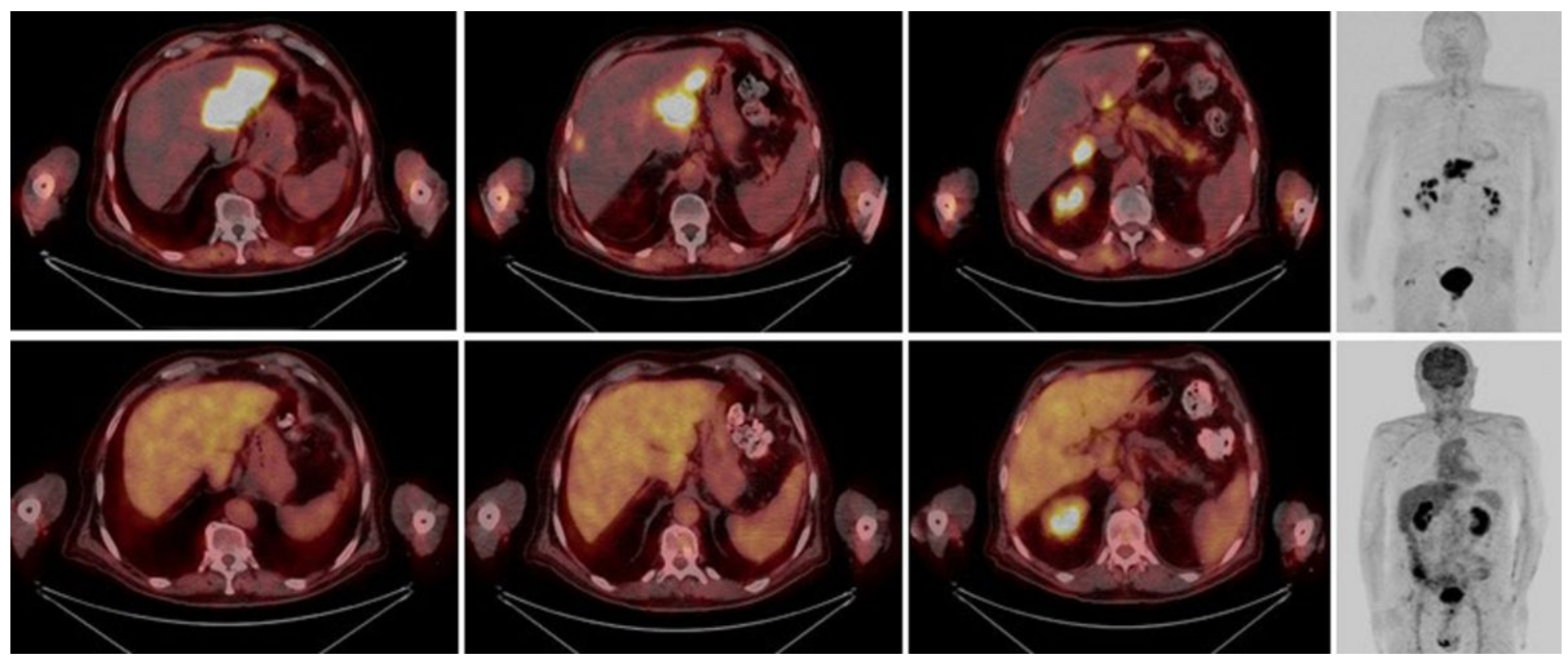

Figure 1 
Transaxial fusion and MIP images of 68Ga-DOTA-FAPI-PET/CT (upper row) and 18FDG-PET/CT [lower row) of an operated patient diagnosed with rectum cancer. 68GaDOTA-FAPI-PET/CT images of the patient show multiple focal increased radiopharmaceutical uptake evaluated in favor of metastases in the liver, while no marked pathologic radiopharmaceutical uptake is observed in the liver on 18FDG-PET/CT images.
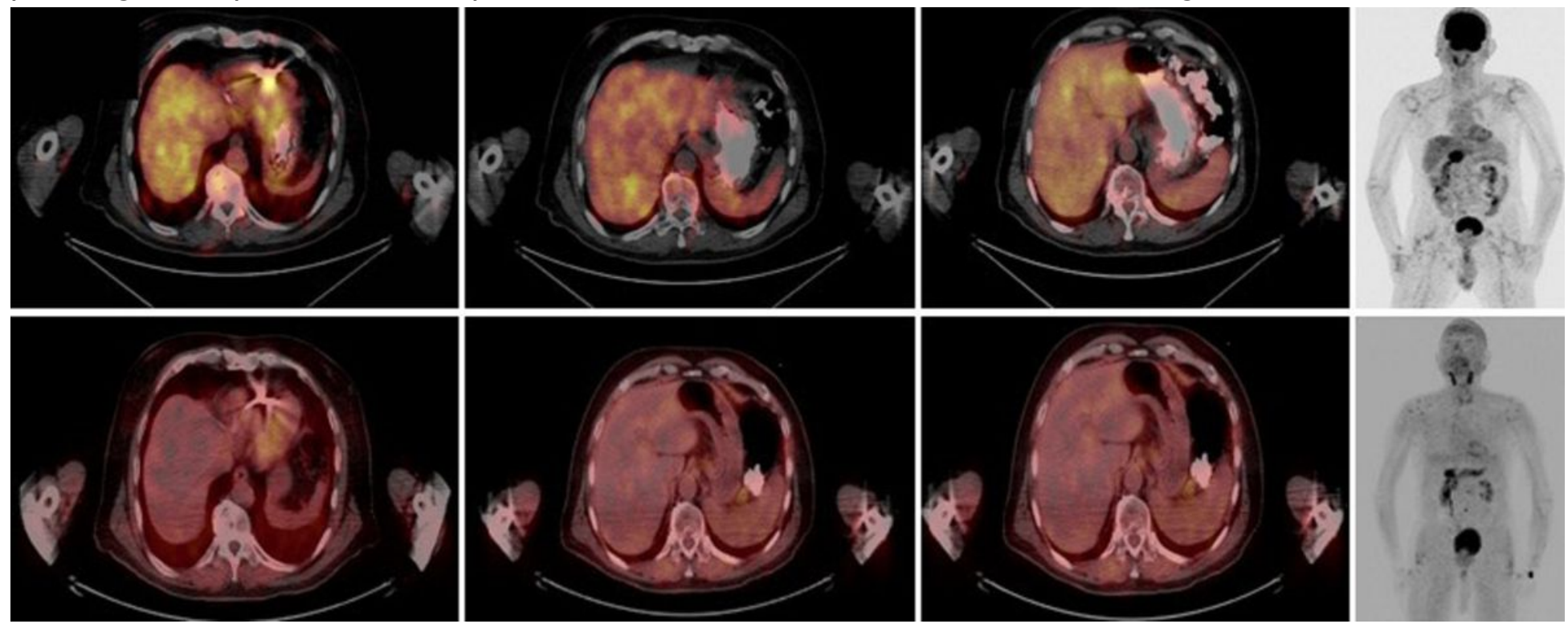

\section{Figure 2}

Transaxial fusion and MIP images of 18FDG-PET/CT (upper row) and 68Ga-DOTA-FAPI-PET/CT (lower row) of a patient diagnosed with gastric cancer. 18FDG-PET/CT images of the patient show small focal radiopharmaceutical uptakes evaluated in favor of metastases in the liver, while no marked pathologic radiopharmaceutical uptake is observed in the liver on 68GaDOTA-FAPI-PET/CT images. 